\title{
To Investigate the Effect of Nerve Growth Factor on Neurons by Establishing the Animal Model of Spinal Cord Injury
}

WEI RAO, W. F. CAO AND Z. J. MEI*

Department of Neurology, Jiangxi Provincial People's Hospital, No. 92 Aiguo Rd, Nanchang 330006, China

\section{Rao et al.: Effect of Nerve Growth Factor on Neurons of Spinal Cord Injury}

To explore the effect of nerve growth factor on neurons by establishing the animal model of spinal cord injury is the main objective. 40 Sprague Dawley rats were stochasticly divided into a model group and a control group of 20 rats. 5 rats in the model group were selected as model group $A$ and the rest were model group B and 5 rats in the control group were selected as control group $A$ and the rest were control group B. Model group A and control group A were treated with tail vein injection of nerve growth factor. The expression of nerve growth factor, vascular endothelial growth factor, glial fibrillary acidic protein and motor function in groups were observed, to observe the effect of nerve growth factor on proliferation and apoptosis of neuron cells. The expression of nerve growth factor in model group was significantly lower than that in control group $(p<0.05)$ and the expression of nerve growth factor in model group $A$ and control group A was higher than that in model group B and control group B group, respectively $(\mathbf{p}<\mathbf{0 . 0 5})$. Basso, Beattie and Bresnahan score in model group was lower than that in model group $A(p<0.05)$. The expression of glial fibrillary acidic protein and vascular endothelial growth factor in model group A was higher than that of model group $(\mathbf{p}<\mathbf{0 . 0 5})$. The proliferation ability of control group was significantly higher than that of model group $(p<0.05)$, the apoptosis rate of model group was higher than control group $(p<0.05)$. Nerve growth factor participates in the occurrence and development of spinal cord injury and is bound up with the proliferation and apoptosis of neuronal cells.

Key words: Spinal cord injury, nerve growth factor, neurons, vascular endothelial growth factor

As the most serious complication of injury of spine, spinal cord injury often gives rise to severe dysfunction of limbs below the injured segment ${ }^{[1]}$. At present, the incidence of spinal cord injury shows a trend of increasing year by year with the economic level of various countries in the world and the development of modern means of transportation and industrial and mining undertakings ${ }^{[2]}$. With the social and economic progress in our country, medical technology has also made great progress ${ }^{[3]}$. Spinal cord injury is a kind of trauma with extremely serious consequences, however, even though the death rate of spinal cord injury has decreased in recent years, the recovery degree of nerve injury is still not ideal ${ }^{[4]}$. According to previous studies and statistics, spinal cord injury not only brings great changes to patients and families, but also brings economic losses and burdens to society far beyond our estimation ${ }^{[5]}$. Therefore, prevention, treatment and rehabilitation of spinal cord injury have become a major topic in the medical field today ${ }^{[6]}$. Previous studies have shown that biomechanical research can help us to understand the mechanism of spinal cord injury and the influence of injury force on biochemical and pathological changes of spinal cord, which can further guide prevention and treatment $t^{[7]}$. However, due to its complex structure, there are relatively few biological researches.

In clinical medical research, there are numerous limitations in selecting human beings as research objects and lots of experimental methods will be limited ${ }^{[8]}$. The use of animal models for experiments is an indispensable means in medical technology ${ }^{[9]}$, which plays a vital role in seeking new and effective treatment

*Address for correspondence

E-mail: meizj0125@163.com 
methods. The irreversibility of neurological deficits caused by spinal cord injury is mainly the result of the difficulty of regeneration of injured neurons ${ }^{[10]}$. As a protein, nerve growth factor (NGF) can be obtained from various animals ${ }^{[11]}$. NGF can regulate the growth and progression of peripheral neurons and maintain survival. It produces a marked effect on regulating the development, growth, differentiation, regeneration and expression of functional characteristics of central and peripheral neurons ${ }^{[12]}$. Therefore, this study aims at exploring the impacts of NGF on neurons by establishing spinal cord injury animal model, thus to provide reference and guidance for future clinical diagnosis and treatment of spinal cord injury.

\section{MATERIALS AND METHODS}

\section{Animal data:}

40 Sprague Dawley (SD) rats of clean grade $6 \mathrm{w}$ old were selected as the experimental subjects. They were bought from Beijing Vital River Laboratory Animal Technology Co., Ltd., with the certificate number of SCXK (Jing) 2016-0011, including half female and half male, weighing (180-220) g. The rats were kept in cages (10 animals in one cage) with normal indoor feeding, light, temperature $(29 \pm 2)^{\circ}$ and humidity of $40 \%-50 \%$. They were stochasticly divided into two groups (model group (MG) and control group (CG)), with 20 rats in each group. The MG was injected with normal saline via tail vein $30 \mathrm{~min}$ to $1 \mathrm{~h}$ after modeling. Five rats in the $M G$ were stochasticly selected as model group A (MGA) and the rest were model group B (MGB). And 5 rats in the CG were stochasticly selected as control group A (CGA) and the rest were control group B (CGB). MGA and CGA were treated by tail vein injection of NGF for $30 \mathrm{~min}$ to $1 \mathrm{~h}$, with NGF 30 $\mu \mathrm{g} / \mathrm{kg} / \mathrm{d}$ and $1 \mathrm{ml}$ of normal saline for $3 \mathrm{~d}$.

\section{Animal modeling:}

SD rats in MG were anesthetized with intraperitoneal injection of $30 \mathrm{~g} / \mathrm{l}$ pentobarbital sodium $(40 \mathrm{mg} / \mathrm{kg}$ ) and then they were immobilized in prone position. The back hairs of the rats were removed, disinfected and treated with surgical drape. Animal models of T10 spinal cord injury were established by Allen's method ${ }^{[13]}$. Firstly, the location of T8 spinous process was determined. Then the skin was incised at the linea mediana posterior. Paravertebral muscles were bluntly separated and supraspinous and interspinous ligaments were removed by eye scissors to expose the $\mathrm{T} 7$ to $\mathrm{T} 9$ spinous processes of rats. T8 vertebral plate was removed by bone rongeur to expose the dura mater. Finally, T8 was clamped by $30 \mathrm{~g}$ vascular clamp for $1 \mathrm{~min}$ and twitches of different degrees might occur in both hind limbs and tail of rats, suggesting that the spinal cord injury model was successfully prepared.

\section{Sample detection:}

The expression of NGF in MG and CG was examined by enzyme-linked immunosorbent assay (ELISA) and Western blot. Then, the expression of NGF in MGA and MGB and of CGA and CGB were detected by ELISA. Rat neurons were taken to detect the impacts of NGF on the proliferation and apoptosis of neurons.

\section{Detection methods:}

ELISA: Blank well, standard sample well and sample to be tested well were set up. S0 standard substance with the concentration of 0 was put into the blank well and 50 $\mu 1$ of standard substance with different concentrations were put into the standard well. In the sample well, 10 $\mu 1$ of sample to be tested was firstly added to it and then added with sample diluent $40 \mu \mathrm{l}$. Nothing was added to the blank well. In addition to the blank wells, 100 $\mu 1$ of horseradish peroxidase (HRP) labeled detection antibody was put into each of the standard wells and the sample wells. The reaction wells were blocked with a sealing plate membrane and incubated in a water bath at $37^{\circ}$ for $65 \mathrm{~min}$. After discarding the liquid, the absorbent paper was patted dry. Each well was filled with washing liquid and allowed to stand for $2 \mathrm{~min}$. The washing liquid was thrown off and the absorbent paper was patted dry. This procedure was repeated 6 times. Substrates A and B were put into each well, $50 \mu 1$ each and incubation was performed at $37^{\circ}$ in the dark for $10 \mathrm{~min}$. The optical density (OD) value of each well was determined at $450 \mathrm{~nm}$ wavelength within $15 \mathrm{~min}$ after adding stop solution $(50 \mu 1)$ to each well and the concentration was calculated.

Western blot detection: Protein was extracted from each group of cultured cells with radioimmunoprecipitation assay (RIPA) buffer according to instructions and the lysate was centrifuged for $20 \mathrm{~min}$ at $10000 \times \mathrm{g}$ to collect the supernatant. Bicinchoninic acid (BCA) assay method (using bovine serum albumin as the standard) was applied for protein concentration determination. The same amount of protein was separated and treated with sodium dodecyl sulfate polyacrylamide gel electrophoresis (SDS-PAGE), membrane transfer and 5 $\%$ skim milk powder sealing at indoor temperature for 1 h. NGF and Glyceraldehyde 3-phosphate dehydrogenase (GAPDH) primary antibody were added and incubated overnight at $4^{\circ}$. Then, HRP labeled goat anti-mouse 
immunoglobulin $\mathrm{G}(\mathrm{IgG})$ secondary antibody was put into it for incubation for $2 \mathrm{~h}$ and the luminescent liquid was applied for exposure and development.

\section{3-(4,5-dimethylthiazol-2-yl)-2,5-diphenyl} tetrazolium bromide (MTT) detection: The extracted rat neuron cells were inoculated into $96-$-well wells with $5 \times 10^{3}$ cells per well and cultured at $37^{\circ}$ in a $5 \% \mathrm{CO}_{2}$ incubator. The plates were taken out at $24 \mathrm{~h}, 48 \mathrm{~h}, 72 \mathrm{~h}$ and $96 \mathrm{~h}$, respectively, added with MTT solution $(5 \mathrm{mg}$ / $\mathrm{ml}$, Sigma-Aldrich) and cultured for $4 \mathrm{~h}$. When MTT solution was removed, dimethylsulfoxide (DMSO) (150 $\mu$ l, Sigma-Aldrich) was put into each well, shaken for $10 \mathrm{~min}$ and the density (OD) at $570 \mathrm{~nm}$ wavelength was assessed using a microplate reader. The experiment was repeated 3 times and the average results were taken.

Flow cytometry: Rat neuron cells were collected and treated according to instruction of fluorescein isothiocyanate (FITC). Cells were collected and centrifuged and incubated at a density of $1 \times 10^{6} / \mathrm{ml}$. Cell suspension $(100 \mu \mathrm{l})$ was incubated with annexin V-FITC $(10 \mu \mathrm{l})$ for $15 \mathrm{~min}$ and propidium iodide (PI) was added for re-staining in dark for $30 \mathrm{~min}$. Flow cytometry was used for detection. In the two-parameter diagram, negative annexin V and PI were normal cells, positive annexin $\mathrm{V}$ were early apoptotic cells and positive annexin $\mathrm{V}$ and PI were late apoptotic cells. Apoptosis rate of cells=percentage of early apoptosis cells+percentage of late apoptosis cells.

Outcome measures: The expression of NGF in MG and CG, MGA and MGB and CGA and CGB were observed. The motor function of rat hind limbs was determined by Basso, Beattie and Bresnahan (BBB) scoring method. Polymerase chain reaction (PCR) was used for determination of the expression of vascular endothelial growth factor (VEGF) and glial fibrillary acidic protein (GFAP) in rat spinal cord and the impacts of NGF on proliferation and apoptosis of neuron cells.

\section{Statistical analysis:}

All statistical analysis of the experimental results was performed by statistical package for the social sciences (SPSS) 24.0 statistical software. All graph results were plotted by Graphpad 8. All the data in this experiment were expressed in the form of mean \pm standard deviation. Comparison between groups adopted t test. Comparison among multiple groups adopted one-way analysis of variance and least significant difference (LSD) back testing. $\mathrm{p}<0.050$ was considered statistically significant.

\section{RESULTS AND DISCUSSION}

NGF expression in the MG and the CG was detected by ELISA and Western blot. The results exhibited that the MG was remarkably lower than the CG, with statistical difference $(p<0.05)$ as shown in fig. 1 .
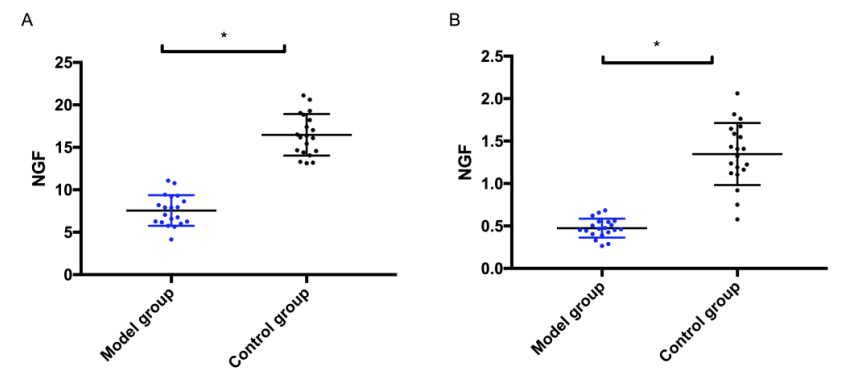

Fig. 1: NGF expression in MG and CG

A: ELISA detection of NGF expression in MG and CG. B: Western blot detection of NGF expression in MG and CG group

ELISA was utilized to measure the expression of NGF in MGA and MGB, CGA and CGB. It could be seen from the result that the NGF expression in MGA was higher than that in MGB ( $<<0.05)$, in CGA was higher than that in CGB $(\mathrm{p}<0.05)$, in CGA was higher than that in MGA $(\mathrm{p}<0.05)$ and in CGB was higher than that in MGB $(\mathrm{p}<0.05)$ as shown in fig. 2.

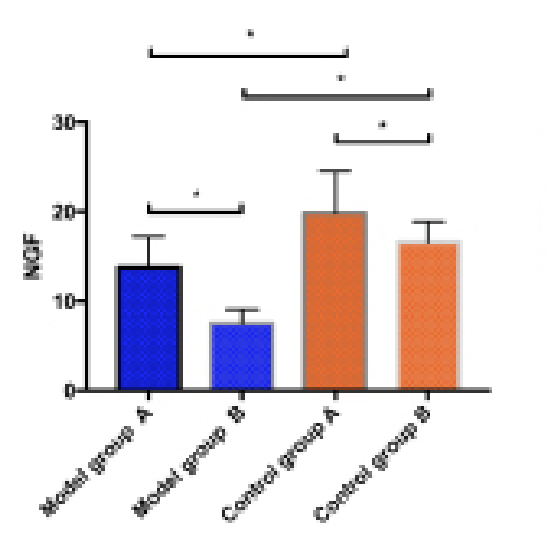

Fig. 2: NGF expression in MGA and MGB, CGA and CGB,

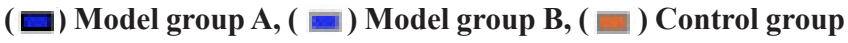
A, ( $\mid=$ ) Control group B,

BBB scoring method was used to evaluate the hind limb motor function of rats. The scores of $M G$ and MGA was found to be decreased after modeling and the scores of MG were lower than that of MGA $(p<0.05)$. After modeling, the score of MGA was higher than that after modeling for $2 \mathrm{~d}(\mathrm{p}<0.05)$, while the score of CGA was higher than that after modeling for $2 \mathrm{~d}(\mathrm{p}<0.05)$ as shown in fig. 3. 


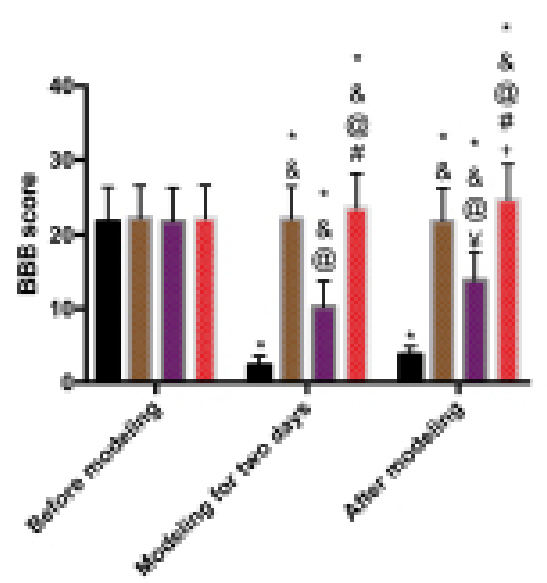

Fig. 3: Score of motor function of hind limbs in rats Note: *indicates comparison with before modeling, ${ }^{8}$ indicates comparison with modeling group, ${ }^{a}$ indicates comparison with CG, "indicates comparison with MGA, ${ }^{\text {indicates comparison }}$ with modeling for $\mathbf{2} \mathbf{d}$, ${ }^{+}$indicates comparison with modeling for 2 d, ( $\mathbf{a})$ Model group A, ( $\mid \mathbf{m})$ Model group B, ( $\mathbf{n}$ ) Control group $\mathbf{A},(=)$ Control group $\mathbf{B}$

VEGF and GFAP protein expression in rats of each group was observed. The results indicated that VEGF expressed highly in the CG and low in the MG. VEGF expression in the MGA was higher than that in the MG $(p<0.05)$, VEGF expression in the CGB was higher than that in the CG. GFAP was expressed low in the CG and expressed high in the MG. VEGF expression in the MGA was lower than that in the MG $(\mathrm{p}<0.05)$ and in the CGA was lower than that in the CG $(\mathrm{p}<0.05)$ as shown in fig. 4.
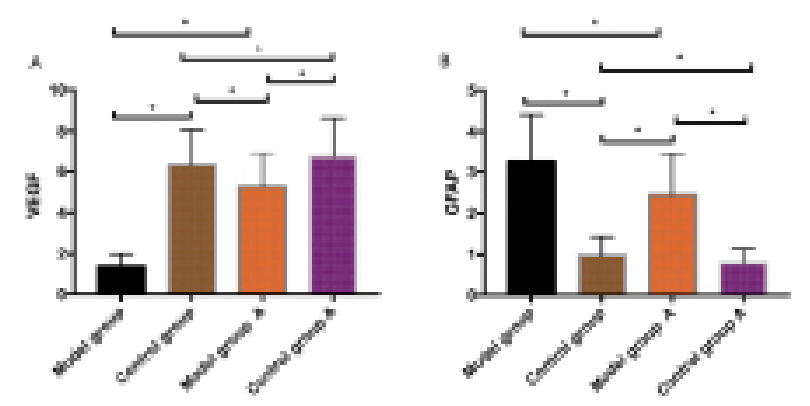

Fig. 4: Expression of VEGF and GFAP protein in rats of each group

A: Expression of VEGF in rats of each group. B: Expression of GFAP in rats of each group, ( $\square)$ Model group A, ( $=$ ) Model group B, ( = ) Control group A, ( $=$ ) Control group B

MTT assay revealed that the proliferation ability of CG was notably higher than the $M G(p<0.05)$, while that of MGA was notably higher than that of MG $(\mathrm{p}<0.05)$. There was no remarkable difference between $\mathrm{CG}$ and CGA ( $>0.05)$ as shown in fig. 5 .

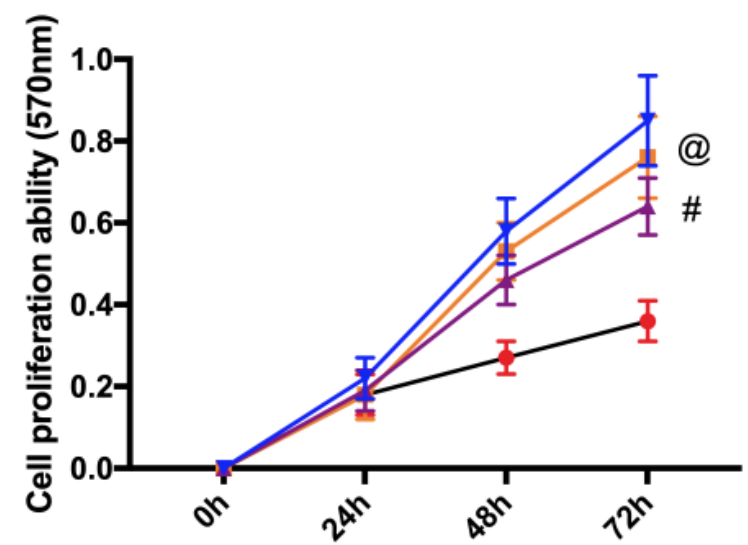

Fig. 5: Neuron cell proliferation

Note: ${ }^{\circledR}$ means comparison between CG and MG, "means comparison between MGA and MG, ( $\rightarrow$ ) Model group A, ( - ) Model group B, $(-)$ Control group A, $(\rightarrow)$ Control group B The apoptosis rate of neuron cells was determined by the aid of flow cytometry and the results revealed that the apoptosis rate of MG was higher than that of $\mathrm{CG}$ $(p<0.05)$ and MGA was lower than that of $M G(p<0.05)$ as shown in fig. 6 .

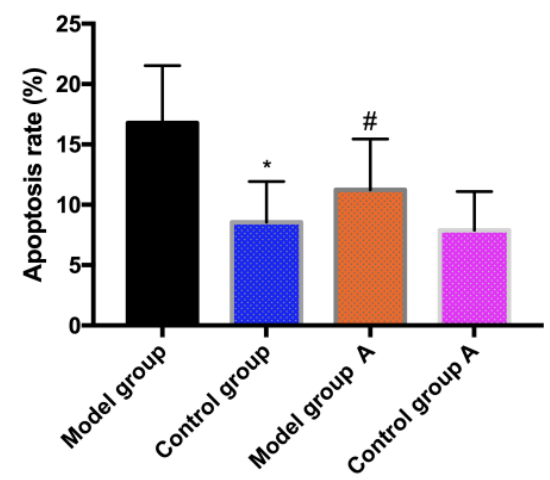

Fig. 6: NGF on neuron cells apoptosis

Note: *indicates the ratio of $\mathrm{CG}$ to $\mathrm{MG}$, "indicates the ratio of MGA to MG, ( $\square$ ) Model group, ( $(\square)$ Control group, ( $\square$ )

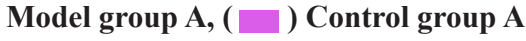

Spinal cord injury is a common clinical neurological injury disease, but its pathological mechanism is relatively complex and there is no ideal treatment method ${ }^{[14]}$. As one of the diseases with the highest disability rate recognized in the society at present, spinal cord injury of patients is extremely easy to lead to dysfunction and loss of labor ability and its burden on patients and society is immeasurable ${ }^{[15]}$. In recent years, with the discovery of growth related factors, a new idea for the treatment of spinal cord injury has been opened ${ }^{[16]}$. NGF is the earliest discovered cell growth regulator and the most deeply studied neurotrophic factor, which is widely used in clinical treatment of central nervous system injury ${ }^{[17]}$. Studies have also shown that NGF protects nerves through various mechanisms and links ${ }^{[18]}$. The 
recovery of neurons is an important factor in the recovery of spinal cord injury ${ }^{[19]}$. Therefore, we carried out a study on the effect of NGF on neurons to provide a reliable and effective reference for the clinical promotion of spinal cord nerve function recovery in the future.

Here, NGF expression in MG and CG was detected by ELISA and Western blot and in MG was lower than that in CG, suggesting that NGF might participate in the occurrence and progression of spinal cord injury. ELISA detected the expression of NGF in MGA and group MGB, CGA and CGB. The results suggested that NGF expression in MGA was higher than that in MGB and in CGA was higher than that in CGB. This result suggested successful modeling and indicated that NGF treatment had a certain recovery effect on neurons of spinal cord injury rats, which could further reduce the level of spinal cord injury in rats. The research conducted by the team of Zhang et al. ${ }^{[20]}$ shows that electroacupuncture can improve neurogenic bladder dysfunction by activating NGF/neurotrophin receptor (TrkA) signal transduction in rat model, which is similar to the results of this study. NGF is one of neurotrophic factors. $\beta$ subunit is the active functional center of NGF, which mainly exists in the cell body, cytoplasm and processes of neurons. It can regulate the development and growth of nerve cells and axons and is expressed in central and peripheral neurons, maintaining the differentiation, development and regeneration of nerve cells $^{[21]}$. We speculated that the expression of NGF in spinal cord injury rats injected with NGF was enhanced when neurons were damaged, which promoted the repair of cell membrane, thus reducing the continuous damage of neurons and accelerating the regeneration of neuron cells. Subsequently, BBB scoring method was used for determination of hind limb motor function of rats. The score of MG was found to be lower than that of MGA, which further confirmed that NGF was beneficial in the improvement and recovery of nerve function and supported the above experimental results. Then VEGF and GFAP proteins in spinal cord were detected. It was found that VEGF showed high expression in the CG and low expression in the MG. VEGF expression in the MGA was higher than that in the MG and in the CGB was higher than that in the CG. GFAP showed low expression in the $\mathrm{CG}$ and high expression in the MG. VEGF expression in the MGA was lower than that in the MG and in the CGA was lower than that in the CG. VEGF is a member of angiogenesis family, which can promote the proliferation of endothelial cells and the generation of capillaries. VEGF also has neuroprotective effect, which can increase the activity and survival rate of neuronal cells and promote axonal regeneration $^{[22]}$. The results suggest that NGF produces a marked effect on the treatment of spinal cord injury. GFAP is an important skeleton protein synthesized by astrocytes. It is generally believed that the up-regulation of GFAP expression represents the degree of astrocyte proliferation $^{[23]}$. However, in this study, GFAP level in MGA was lower than that in MG, indicating that NGF has a reducing effect on the activity of astrocytes and reduces the level of spinal cord injury in rats. In order to further determine the effect of NGF on spinal cord injury, we executed rats and took rat neuron cells to analyze the effect of NGF on them. And we found that the proliferation ability of the CG was remarkably higher than that of the MG, while the proliferation ability of the MGA was significantly higher than that of the MG, the apoptosis rate of the MG was higher than that of the CG and the apoptosis rate of the MGA was lower than that of the MG. This suggests that the regeneration ability of neuron cells in group A of MG treated with NGF was enhanced, while normal rats treated with NGF could also promote the proliferation of neuron cells to a certain extent. It shows that NGF has a good effect on promoting the regeneration of neuron cells without side effects and may also have certain applicable value for normal people, which is consistent with our above experimental results. According to this result, we speculated that NGF might improve all diseases related to neuronal cell injury to some extent, which requires us to carry out more experiments for analysis and confirmation. We will take corresponding measures as soon as possible to analyze the deeper influence mechanism of NGF on neuronal cells.

There are still deficiencies in this experiment. Due to the short experimental period, we cannot evaluate whether NGF has any influence on the long-term prognosis of spinal cord injury rats. In this experiment, neuron cells were not purchased for in vitro experimental analysis, so it is not clear how NGF affects neuron cells. And no human trials have been conducted and it is uncertain how NGF will behave in human spinal cord injury patients. It is not excluded that there may be some differences with animal models and a large number of clinical trials are needed to confirm the application in human body. We will conduct a more in-depth and comprehensive analysis of the deficiencies of this experiment to obtain more representative experimental results for clinical reference.

To sum up, NGF participates in the occurrence and progression of spinal cord injury and is bound up 
with the proliferation and apoptosis of neuronal cells. Targeted treatment with NGF may be a new direction for spinal cord injury in the future.

\section{Conflicts of interest:}

The authors declared no conflict of interest.

\section{REFERENCES}

1. O'Shea TM, Burda JE, Sofroniew MV. Cell biology of spinal cord injury and repair. J Clin Invest 2017;127(9):3259-70.

2. Ahuja CS, Wilson JR, Nori S, Kotter MR, Druschel C, Curt A, et al. Traumatic spinal cord injury. Nat Rev Dis Primers 2017;3(1):1-21.

3. Ren Y, Ao Y, O'Shea TM, Burda JE, Bernstein AM, Brumm $\mathrm{AJ}$, et al. Ependymal cell contribution to scar formation after spinal cord injury is minimal, local and dependent on direct ependymal injury. Sci Rep 2017;7(1):1-6.

4. Assinck P, Duncan GJ, Hilton BJ, Plemel JR, Tetzlaff W. Cell transplantation therapy for spinal cord injury. Nat Neurosci 2017;20(5):637-47.

5. Dias DO, Kim H, Holl D, Solnestam BW, Lundeberg J, Carlen $\mathrm{M}$, et al. Reducing pericyte-derived scarring promotes recovery after spinal cord injury. Cell 2018;173(1):153-65.

6. Wagner FB, Mignardot JB, Le Goff-Mignardot CG, Demesmaeker R, Komi S, Capogrosso M, et al. Targeted neurotechnology restores walking in humans with spinal cord injury. Nature 2018;563:65-71.

7. Tran AP, Warren PM, Silver J. The biology of regeneration failure and success after spinal cord injury. Physiol Rev 2018;98(2):881-917.

8. Lui YF, Ip WY, Shad M, Chan HY. Chicken proximal interphalangeal joint as an animal model for human finger joint prosthesis evaluation. J Orthop 2020;20:105-10.

9. Sorzano CO, Parkinson M. Statistical experiment design for animal research; 2019.

10. Keefe KM, Sheikh IS, Smith GM. Targeting neurotrophins to specific populations of neurons: NGF, BDNF, and NT-3 and their relevance for treatment of spinal cord injury. Int $\mathrm{J}$ Mol Sci 2017;18(3):548.

11. Denk F, Bennett DL, McMahon SB. Nerve growth factor and pain mechanisms. Annu Rev Neurosci 2017;40:307-25.

12. Song Z, Wang Z, Shen J, Xu S, Hu Z. Nerve growth factor delivery by ultrasound-mediated nanobubble destruction as a treatment for acute spinal cord injury in rats. Int $\mathrm{J}$ Nanomedicine 2017; 12:1717.

13. Liu R, Wang W, Wang S, Xie W, Li H, Ning B. microRNA-21 regulates astrocytic reaction post-acute phase of spinal cord injury through modulating TGF- $\beta$ signaling. Aging 2018;10(6):1474.
14. Manohar A, Foffani G, Ganzer PD, Bethea JR, Moxon KA. Cortex-dependent recovery of unassisted hindlimb locomotion after complete spinal cord injury in adult rats. eLife 2017;6:e23532.

15. Burke D, Fullen BM, Stokes D, Lennon O. Neuropathic pain prevalence following spinal cord injury: A systematic review and meta-analysis. Eur J Pain 2017;21(1):29-44.

16. Li R, Wu Y, Zou S, Wang X, Li Y, Xu K, et al. NGF attenuates high glucose-induced ER stress, preventing schwann cell apoptosis by activating the PI3K/Akt/GSK3 $\beta$ and ERK1/2 pathways. Neurochem Res 2017;42(11):3005-18.

17. Cheng YY, Zhao HK, Chen LW, Yao XY, Wang YL, Huang $\mathrm{ZW}$, et al. Reactive astrocytes increase expression of proNGF in the mouse model of contused spinal cord injury. Neurosci Res 2020;157:34-43.

18. Mili B, Das K, Kumar A, Saxena AC, Singh P, Ghosh S, et al. Preparation of NGF encapsulated chitosan nanoparticles and its evaluation on neuronal differentiation potentiality of canine mesenchymal stem cells. J Mater Sci Mater Med 2018;29(1):13.

19. Wang Z, Zhou L, Zheng X, Chen G, Pan R, Li J, et al. Autophagy protects against PI3K/Akt/mTOR-mediated apoptosis of spinal cord neurons after mechanical injury. Neurosci Lett 2017;656:158-64.

20. Zhang T, Yu J, Huang Z, Wang G, Zhang R. Electroacupuncture improves neurogenic bladder dysfunction through activation of NGF/TrkA signaling in a rat model. J Cell Biochem 2019;120(6):9900-5.

21. Rocco ML, Soligo M, Manni L, Aloe L. Nerve growth factor: early studies and recent clinical trials. Curr Neuropharmacol 2018;16(10):1455-65.

22. Rumney RM, Lanham SA, Kanczler JM, Kao AP, Thiagarajan $\mathrm{L}$, Dixon JE, et al. In vivo delivery of VEGF RNA and protein to increase osteogenesis and intraosseous angiogenesis. Sci Rep 2019;9(1):1-10.

23. Griffin JM, Fackelmeier B, Fong DM, Mouravlev A, Young D, O'Carroll SJ. Astrocyte-selective AAV gene therapy through the endogenous GFAP promoter results in robust transduction in the rat spinal cord following injury. Gene Ther 2019;26(5):198-210.

This is an open access article distributed under the terms of the Creative Commons Attribution-NonCommercial-ShareAlike 3.0 License, which allows others to remix, tweak, and build upon the work non-commercially, as long as the author is credited and the new creations are licensed under the identical terms

This article was originally published in a special issue,

"Therapeutic Perspectives in Biomedical Research and Pharmaceutical Sciences and their Nursing Methods"

Indian J Pharm Sci 2021:83(4)Spl issue "102-107" 